JOURNAL OF

APPLIED

CRYSTALLOGRAPHY

ISSN 1600-5767

Received 9 February 2016

Accepted 9 February 2016

Keywords: neutron reflectivity data analysis; computer programs

\section{Aurore: new software for neutron reflectivity data analysis. Corrigendum}

\author{
Yuri Gerelli*
}

Institut Laue-Langevin 71, avenue des Martyrs, 38000, Grenoble, France. *Correspondence e-mail: gerelli@ill.fr

An incorrect statement in the paper by Gerelli [J. Appl. Cryst. (2016), 49, 330$339]$ is corrected.

An incorrect statement in the paper by Gerelli (2016) is corrected. On p. 332, $\$ 2.1$ it is stated that negative reflectivity values are discarded by default in the Aurore software because they are not physical. The statement is incorrect because, although reflectivity is a positive-definite quantity, for the case of background-subtracted data the reflectivity values can fluctuate around zero. This occurs especially if the reflectivity values are very small compared to the background. Ignoring systematically negative values could bias fits under these circumstances.

\section{References}

Gerelli, Y. (2016). J. Appl. Cryst. 49, 330-339.

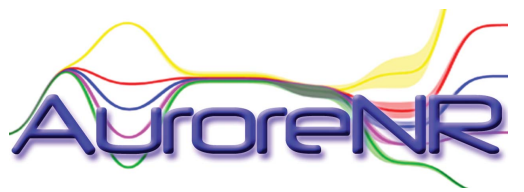

(C) 2016 International Union of Crystallography 